\title{
Human Papillomavirus Genotyping among Different Cervical Smears in Duhok/Iraq
}

\author{
Intisar Salim Pity ${ }^{1}$, Hanaa Mohammed $\mathrm{Abdo}^{2}$, Amer Abdalla Goreal ${ }^{2 *}$
}

\begin{abstract}
Background/Objective: Cervical cancer ranks the second among the most common gynecologic cancers. This study was established to determine the distribution of cervical HPV genotypes among different Pap readings in Duhok/Iraq. Methods: Between January and September-2016, HPV-DNA was tested in 64 women. Genotyping was carried out by the hybridization reverse blot technique. Cervical smears were taken, performed by ThinPrep technique and stained by Pap stain. Results: Twenty six (40.6\%) cases were positive for HPV, 12 (46.2\%) in normal and 14 (53.8\%) in abnormal Pap smears. 39 (19 high-risk and 16 low-risk) genotypes were identified. The high risk group comprised 6 HPV16, 4 HPV18, 2 HPV66, 2 HPV52, 2 HPV39, 1 HPV56, 1 HPV31 and 1 HPV45. The 16 low risk strains encompassed 4 HPV6 strains, 4 HPV71, 2 HPV54 and 2 HPV83, HPV11, HPV61 HPV84, and HPV62. Mixed infections were described in 4 women (6.25\%), limited to the NILM, ASC-US and LSIL smears. They included variable admixtures of 7 high risk genotypes, HPV39 (both copies), HPV66 (both copies), HPV52, HPV31, HPV45 and 6 low risk strains: HPV83, HPV6, HPV11, HPV54, HPV62 and HPV71. Conclusions: The higher frequency of HR-HPV than the LR-HPV with identification of 4 mixed cases indicates that our women are at risk of developing cervical cancer. Detection of HR-HPV in NILM and ASC-US smears with restriction of some strains to these 2 categories highlights the great value of HPV genotyping as a surrogate test to pick up unscreened women at risk of developing cervical malignancy particularly when a proper screening program is absent.
\end{abstract}

Keywords: HPV- Genotypes- Pap smear

Asian Pac J Cancer Prev, 20 (7), 2059-2064

\section{Introduction}

Cervical cancer remains a public health problem with a disproportionate occurrence globally. It ranks the second among the most common gynecologic cancers among women and accounts for about $13 \%$ of female cancers (Sauvageau et al., 2007; American Social Health Association, 2012). In Iraq, the region Duhok- belongs to, cervical cancer is not common and forms about $1 \%$ of all female cancer cases (Othman etal., 2007; Iraqi cancer registry (WHO) 2012), however women at risk for developing this cancer forms about 10.7 million cases (Fact Sheet, 2016). It is accepted that invasive cervical cancer is considered as a preventable disease as it is preceded by effectively treatable, long standing preneoplastic and preinvasive states (Schiffman and Wentzensen, 2013; Vink et al., 2013; Nayar and Wilbur, 2015). Molecular epidemiologic evidences indicate that persistent infection with certain HPV strains have been implicated in most invasive and preinvasive cervical cancers and, therefore. The diversity of HPV spectrum and the high incidence of mixed infections make it essential to establish reliable methods for identification of the various
HPV genotypes, not only for epidemiologic studies but also for patient vaccination and management (Clifford etal., 2005; Natphopsuk et al., 2013). Limited studies have been conducted regarding identification of HPV in cervical cytologic samples in Duhok-Kurdistan-Iraq (Pity et al., 2012; Pity, 2013). However, to the best of our knowledge, no data is available regarding HPV-genotype distribution among variable cervical smear readings in this particular region. Given the fact that in our molecular centers, technology developments for identification of different genotyping are in progress, we found that a work on HPV genotype distribution in different Pap smear readings should be performed in Duhok-Kurdistan/Iraq. This study is an attempt to determine HPV genotypes among Duhok women and to identify the distribution of high and low risk HPV strains in Pap smear readings.

\section{Materials and Methods}

Study population

Sixty four eligible women (17-60 years; mean: 34) who consented to join this study after understanding its

${ }^{1}$ Department of Pathology, ${ }^{2}$ Department of Microbiology, College of Medicine, University of Duhok, Iraq. *For Correspondence: amer.goreal@uod.ac 
nature, were recruited. These women attended Duhok Public Cytologic Centers of Azadi Teaching Hospital and VIN Private Laboratory, from $28^{\text {th }}$ November-2015 to $21^{\text {st }}$ September-2016. Ethical approval to the study was obtained from the Medicine Research Committee Ethics, College of Medicine-University of Duhok. From each woman, 2 cervical samples were taken with a sterilized cytobrushes, one for genetic study and the other for cytologic examination (Pap smear).

\section{HPV_DNA extraction and genotyping}

For HPV-DNA identification and genotyping, full turn cervical brushing was performed in one direction and withdrawn carefully without causing any bleeding. Brushes were rinsed in buffer tubes (containing specific medium for transporting and storing) which were recapped and sealed tightly, labelled with unique ID, date and time of collection. Samples were stored at $2-8^{\circ} \mathrm{C}$ to be processed within one week. The material then was taken onto liquid media that enable concurrent PCR detection of high and low risk HPV. Extraction of DNA from the cervical samples in transporting medium was performed by using The QIAamp Mini Kit (Qiagen, Hilden, Germany). The extraction steps were performed according to the manufacturer's instruction. Genotyping was tested through the hybridization reverse blot technique (Opegen by operon /Spain) according to manufacturer's instructions using high and low human papilloma strip kits that allowed identification of 37 genital HPV-DNA (High risk: 16, 18, $26,31,33,35,39,45,51,52,53,56,58,59,66,68,69$, 73 and 82; Low risk: 6, 11, 40, 42, 43, 44, 54, 61, 62, 67, $70,71,72,74,81,83,84$ and 91). Standard test reference strips with their control positive and negative lines were available for each genotype of the targeted HPV and interpretation of results was undertaken by alignment of the black and red lines of strips with the lines on the chart and then identifying the HPV genotype-bands according to their position on the strip.

Pap smears were taken from the transformation zone by using specific cytobrushes which were immediately placed in methanol containing vials of Thin Prep transport media. Samples were transferred onto numbered microscopic glass slides and stained with Pap stain. Cytological reports were formulated according to the Bethesda System (Verma et al., 2014; Nayar and Wilbur, 2015).

\section{Statistical Analysis}

Descriptive statistics were used to summarize demographic variables. Independent t-test, Fisher's Exact test and chi-squared $\left(\chi^{2}\right)$ test were used for testing the categorical parameters, and differences at the level of $\mathrm{p} \leq 0.05$ were considered as statistically significant.

\section{Results}

Twenty-six $(40.6 \%)$ cases were positive for HPV positive which comprised 16 (25\%) positive HR-HPV (12 pure and 4 mixed \} and 14 (21.9\%) LR-HPV (10 pure and 4 mixed). The highest HPV positivity, 20 (76.9\%), for both high and low risk strains, was at 30-49 year-age and at intermediate level of education and above. However, the differences didn't reach the level of significance $(\mathrm{p}=0.182$ and $\mathrm{p}=0.255$ respectively).

Human Papilloma Virus Genotypes and Pap smear Results

In smears of the 26 positive women, 35 five (19 high-risk and 16 low-risk) HPV genotypes were identified. The viral DNA positivity was identified in $12(38.7 \%)$ negative (NILM) smears and 17 (51.5\%) cytologically abnormal cases. HR-HPVs were found to be significantly high among the LSIL group with $p<0.001$ "As shown in Figure 1".

Of the 19 HR-HPV genotypes, HPV16 was the most frequent genotype $(n=6)$ detected in 3 LSILs, 2 in NILMs and 1 SCC. The second HR=-HPV was HPV18, $(n=4)$, identified in 3 SCC and 1 HSIL. There were 2 HPV66 and 2 HPV39, described as part of mixed infection, one of each in NILM and in ASC-US. Two HPV52 were detected, one as mixed infection in ASC-US while the other was detected alone in NILM. Other 2 HR-HPV genotypes (HPV31 and HPV45) were described in a single LSIL Pap smear. The last HR copy (HPV56) was identified in ASCH "As shown in Figure 2".

The 16 LR-HPV genotypes encompassed 4 HPV6, 3 in NILMs (including a single copy found as part of mixed infection) and one in LSIL. Four HPV71 were

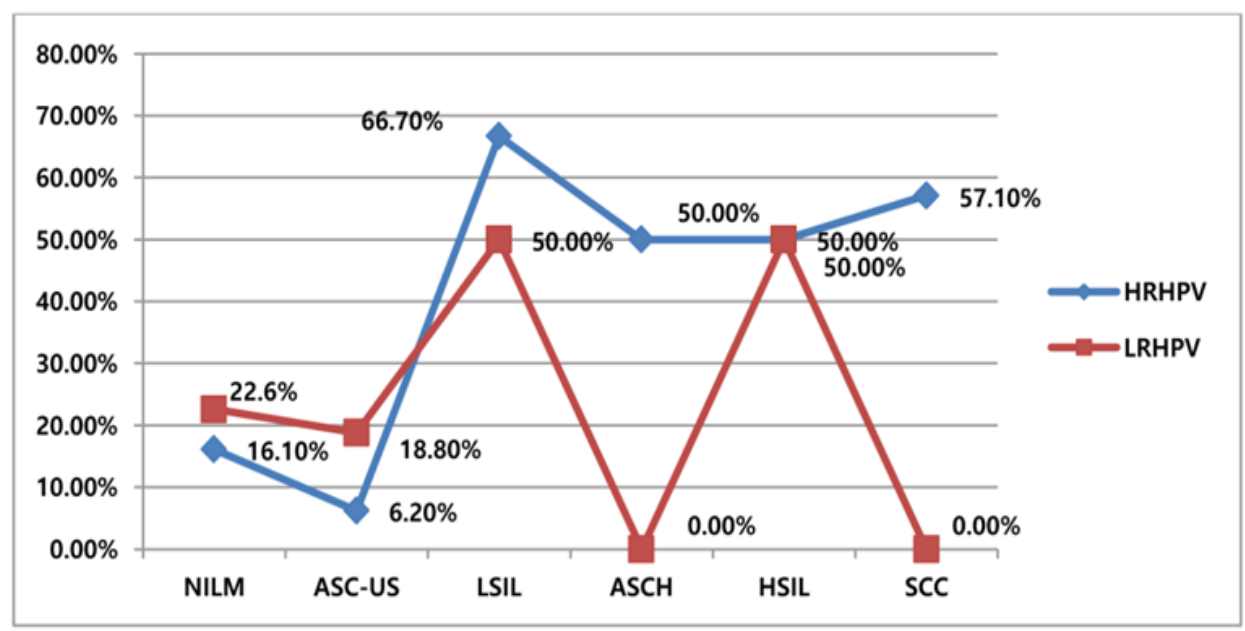

Figure 1. Percentage Distribution of HPV by Pap Results, $\chi^{2}$ Test Used, $p<0.001$. 
Table 1. Frequency of HPV in Different Countries

\begin{tabular}{|c|c|c|c|c|}
\hline Authors & "No of cases" & "HPV frequency in negative smears" & "HPV frequency in positive smears" & Country \\
\hline Current study, 2017 & 64 & $38 / 70 \%$ & $54 / 50 \%$ & Duhok,-Iraq \\
\hline Donkoh, $2016^{14}$ & 593 & $35 / 70 \%$ & $62 / 90 \%$ & Ghana \\
\hline Faik et al, $2015^{20}$ & 856 & $9 / 17 \%$ & $25 \%$ & Baghdad-Iraq \\
\hline Ismail et al, $2014^{21}$ & 57 & $0 \%$ & $71 \%$ & Erbil,Kurdistan-Iraq \\
\hline AL-Awadhi et al, $2013{ }^{22}$ & 326 & $36 / 80 \%$ & $63 / 20 \%$ & Kuwait \\
\hline Derese, 2010 & 360 & $59 / 50 \%$ & $40 / 50 \%$ & Ethiopia \\
\hline
\end{tabular}

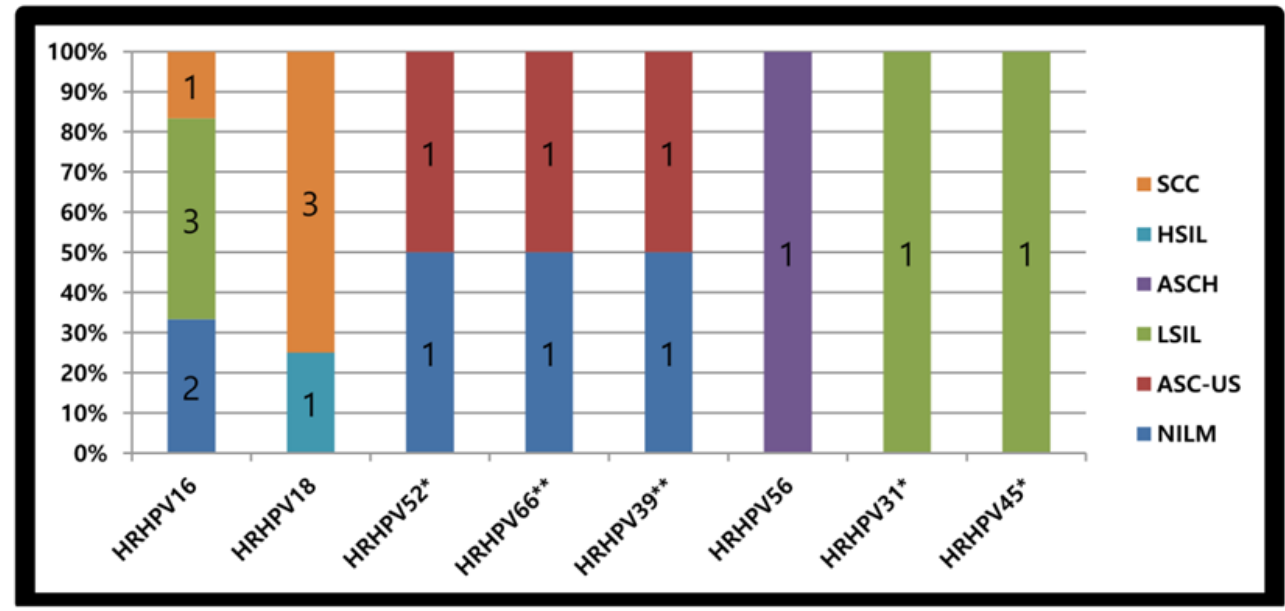

Figure 2. HR-HPV (HRHPV) Genotype Distribution with Pap Results. *, One viral strain detected as part of mixed infection. $* *$, Two viral strains detected as part of mixed infection.

detected, 2 in ASC-US, one in HSIL and one as part of mixed infection in a NILM. There were 2 HPV54, one identified alone in a single LSIL while the other as part of mixed infection in NILM. Two copies of HPV83 were described in NILMs, one alone and one as part of mixed infection. The remainders $(n=4)$ comprised one HPV11 identified as part of mixed infection in a LSIL, one HPV61 and one HPV84 detected separately in 2 NILMs. The last single HPV62 was described mixed with others in ASC-US "As shown in Figure 3".

\section{Mixed HPV Infection}

Multiple HPV infections were described in four women $(6.25 \%)$ limited to the NILM $(n=2)$, ASC-US $(n=1)$ and
LSIL ( $\mathrm{n}=1)$. They included variable admixtures of 7 high risk genotypes which comprised HPV39 (both copies), HPV66 (both copies), HPV52, HPV31, HPV45 and 6 low risk strains which included HPV83, HPV6, HPV11, HPV54, HPV62 and HPV71.

\section{Discussion}

Epidemiological studies have declared the extent of HPV infection burden among various clinical and ethnic populations (Schiffman and Wentzensen, 2013; Vink et al., 2013; Nayar and Wilbur, 2014; Donkoh, 2016; Megia et al., 2016). Iraq anticipates an unusually high prevalence of cervical HPV owing to the lack of organized

Table 2. Frequency of HPV Genotypes in Different Countries

\begin{tabular}{|c|c|c|c|c|}
\hline Authors & "No of cases" & HPV genotypes & HPV genotypes & Country \\
\hline Current study, 2017 & 64 & $\begin{array}{l}\text { "HPV16(31.6\%) } \\
\text { HPV18(21.1\%)" }\end{array}$ & & Duhok,-Iraq \\
\hline Megia et al, $2016^{15}$ & 164 & $\begin{array}{l}\text { "HPV16(41.8\%) } \\
\text { HPV54(30.5\%)" }\end{array}$ & & Ecuador \\
\hline Faik et al, $2015^{20}$ & 856 & $\begin{array}{l}\text { "HPV33(18.60\%) } \\
\text { HPV35(18.60\%)" }\end{array}$ & & Baghdad-Iraq \\
\hline AL-Awadhi et al,2013 22 & 326 & HPV16(24.3\%) & HPV11(13.8\%) & Kuwait \\
\hline Natphopsuk et al, $2013^{9} *$ & 396 & $\begin{array}{l}\text { "HPV16(56.2\%) } \Omega \\
\operatorname{HPV} 58(17.8 \%) \Omega "\end{array}$ & $\begin{array}{c}\text { "HPV } 58(46.4 \%) \Omega \\
\operatorname{HPV} 16(39.3 \%) \Omega "\end{array}$ & Thailand \\
\hline $\mathrm{H}$ amlin-Douglas, $2008^{17}$ & 300 & $\begin{array}{l}\text { "HPV16(5.6\%) } \\
\text { HPV31(3.6\%)" }\end{array}$ & $\begin{array}{l}\text { "HPV -61(3.6\%) } \\
\text { HPV -84(3.1\%)" }\end{array}$ & Nunavik/Canada \\
\hline Duvlis et al, $2001^{26}$ & 190 & $\begin{array}{l}\text { "HPV16(28.4\%) } \\
\text { HPV31(12.1\%)" }\end{array}$ & HPV766(7.9\%) & Macedonia \\
\hline
\end{tabular}




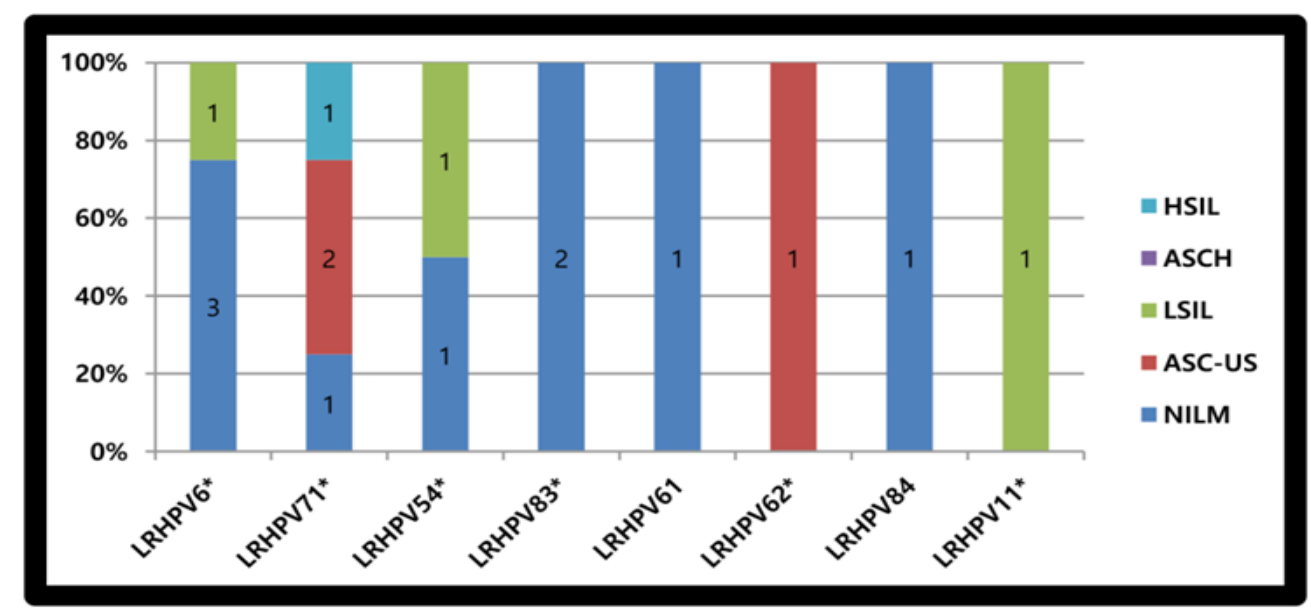

Figure 3. Low Risk HPV (LRHPV) Genotype Distribution with Pap Results. *, One viral strain detected as part of mixed infection.

vaccination program in place. However, in the current study, we detected HPV in $40.6 \%$ of cervical smears. This result falls in the middle of the wide range rates (9.8\%-86\%) worldwide including Iraq and neighboring countries (Adler et al., 2008; Hamlin-Douglas, 2008; Donkoh, 2016; Megia et al., 2016; Abdelbadiaa et al., 2016). However, the HR-HPV frequency (25\%) was a little bit higher than that of a previous large, 8-year follow up, study conducted by Pity et al., (2012) on 10,338 women in the same region where the frequency was $18 \%$. This higher frequency may be explained by the recently used high quality hybridization reverse blot technique. As well, it is higher than what has been reported by Hamlin-Douglas (2008) in his work on 300 Canadian women (20.4\%) (Hamlin-Douglas, 2008). The lack of organized vaccination and screening programs in our country in contrast to the well-established one among Canadian women may reflect their relatively lower rates of HR-HPV (Hamlin-Douglas, 2008). Contrarily, this finding is lower than what had been reported in Africa among 360 Ethiopian and 60 Egyptian women, 78\% and 53\% respectively (Derese, 2010; Abdelbadiaa et al., 2016). Here we can infer that the relatively lower HR-HPV frequency in Kurdistan women may reflect their lower sexual activity and, thus, lower prevalence of HPV infection than the African's. In fact all our participants were legally married women and denied any illegitimate sex owing to the tight constraints of the closed Middle Eastern community with its conservative socio-sexual norms and religious habits.

Amajor strength of the current study is the availability of hybridization reverse blot technique for HPV genotyping and the concurrent Pap smear results for all tested women making it possible to investigate the distribution of low and high risk HPV among different cervical cytologic changes. HPV DNA was identified in $38.7 \%$ of negative (NILM) smears and $54.5 \%$ of cytologically abnormal cases. Data related to the presence of HPV among women with and without cytologic abnormalities show diverse results "As shown in Table 1". This position, however, is contentious since different studies have used different assay methods with variable targeting primers or antibodies and, thus, give overall different mean values.
Therefore, biases should be expected and, even, may not reflect the actual general counts.

High risk HPVs in the current study were observed in $16.1 \%$ of NILM Pap smears, which fall near the lower third of the worldwide ( $0 \%-60 \%$ ) range rates (Clifford et al., 2005; Bosch et al., 2008; Pity et al., 2012; Al-Awadhi et al., 2013; Pity 2013; Faik et al., 2015; Donkoh, 2016). Unmasking the burden of HR-HPV in NILM smears is notorious because of their effective ability to transform normal cell phenotype into a malignant one. Therefore, detection of HR-HPV among NILM cases highlights the high proportion of misdiagnosed cases when depending on morphologic viral changes alone. More seriously, it ascertains the fact that HPV infection remains silent for uncertain period before a serious lesion will develop and many patients after being reassured by negative Pap smears do not use to seek any medical checkup unless they develop signs and symptoms which might be life threatening at one time (Jeney, 2008; Schiffman and Wentzensen, 2013; Schiffman et al., 2015; Donkoh, 2016). The 3-year cumulative risk of CIN3 observed among Californian's women with NILM, using Hybrid Capture 2, was found to be $4.6 \%$. This risk necessitates the crucial HPV testing in all married women and gives the HPV testing a priority to Pap smearing in developed countries (Jeney, 2008; Schiffman et al., 2015). Therefore, women with HR-HPV positive smears should be referred for continuous follow up Pap smearing to pick up any cytologic progression.

Among cytologically abnormal smears, interestingly there was an obvious rising of HR-HPV rates with lesion severity with a strikingly high frequency contributed to LSILs (66.7\%). Although Al-Awadhi et al., (2013) also reported increasing HR-HPV with lesion severity among Kuwait women, however our HR-HPV burden rates among cytologically abnormal smears are considered low when compared to what has been reported among unselected Ethiopians and unscreened Kuwait's women where HR-HPV accounted for more than $95 \%$ of SCC and HSILs (Derese, 2010; Al-Awadhi et al., 2013). This may be attributed to the behavioral profile of the population studied and the method/technique applied for viral detection (Clifford et al., 2005; Al-Awadhi et al., 2013; 
Abdelbadiaa et al., 2016). Consistent with the current study results, Derese failed to identify any LR-HPV among carcinoma cases (Derese, 2010).

Of the 19 HR-HPV genotypes identified in this study, despite the fact that HPV 16 and HPV18 formed the most frequent (31.6\% and $21.1 \%$ respectively), other HR-HPV strains were identified included HPV52, HPV66, HPV39, HPV56, HPV31 and HPV45. In Western Asia region, where Iraq belongs to, about $2.3 \%$ of general women population is estimated to harbor cervical HPV-16/18 infection at a given time, and $72.4 \%$ of invasive cervical cancers are attributed to HPVs 16 or 18 (Clifford et al., 2005). In Baghdad/Iraq, HPV33, HPV35, HPV56, HPV39 and HPV52 were found to be more common than HPV16 and HPV18 (Faik et al., 2015). It is worth mentioning here that the effect of the vaccine available in some local centers has not yet been validated and the available vaccines do not involve HPVs other than HPV16/18. The attributed HPV prevalence and genotype distribution in cervical specimens show an evident divergence "As shown in Table 2". This heterogeneity is mainly attributed to the geographic variations, race and the genetic make-up of individuals in addition to the age group examined and health status of the sampled women. The viral lineage and testing technology used and the DNA region analyzed may also contribute to these rate divergence (Duvlis et al., 2001; Clifford et al., 2005; Natphopsuk et al., 2013; Donkoh, 2016; Megia et al., 2016).

Mixed or co-infection with more than one HPV genotypes is currently arousing much research interest. Despite the fact that it does not necessarily reflect persistence of all the existent strains, this phenomenon is common in a vast fraction of HPV-positive women (Faik et al., 2015; Donkoh, 2016). The proportion of co-infection with multiple HPV genotypes in the present study, $4(15.3 \%)$, is much lower than what has been reported in the literature, 20\%-82\% (Clifford et al., 2005; Adler et al., 2008; Donkoh, 2016). The discrepancies in the rates of mixed HPV infections can be attributed to diversity of populations studied and the restriction of other studies to women at reproductive age which are expected to be sexually more active and, thus, harbor more mixed infections. It is noteworthy that the current study included also older women who may have lower rates of multiple infections. In contrast, the present study is more or less parallel to the study conducted (17.9\%) in Baghdad-Iraq (Faik et al., 2015). The 4 mixed cases were identified in 2 NILM smears, 1 ASC-US and 1 LSIL. In our series, higher grade cervical lesions (HSIL, SCC and even $\mathrm{ASCH}$ ) tended to harbor single (high or low risk) rather than multiple strains. This finding collaborates with that of other researchers who denied any association of mixed HPV infections with the malignant transformation and even might not constitute higher risk factor for the development of high-grade cervical intraepithelial lesions (Levi et al., 2002).

The inherent limitations of this study included a small sample size and the tested sample comprised included only legally married women seeking gynecologic problems which might not represent the general population.

In conclusion, the higher frequency of HR-HPV than the LR-HPV with identification of 4 mixed cases a strongly indicates that our women are at risk of developing cervical cancer and thus vaccination program has to be launched. HPV 16/18 genotypes contributed the highest proportion of the overall HR-HPV with rising frequency among increasing severity of cervical cytologic abnormality. Detection of HR-HPV in NILM and ASC-US Pap smears with restriction of some strains to these 2 categories highlights the great value of HPV genotyping as a surrogate virology test to pick up unscreened women at the risk of developing cervical cancer particularly in absence of a proper screening program.

Ethical approval to the study was obtained from the Medicine Research Committee Ethics, College of Medicine-University of Duhok.

\section{Acknowledgements}

We would like to express our gratefulness to the department of microbiology at the college of Medicine in Duhok University and our thanks to Dr. Jasim Abdo for his technical support.

\section{Conflict of interest}

The authors would like to declare that there was no conflict of interest in this research.

\section{References}

Abdelbadiaa M, Shaker OG, Hosni HN, Khalifa S, Shazly AF (2016). Human papillomavirus (HPV) in Egyptian females: study by cytology, histopathology, colposcopy and molecular diagnosis of high risk types. Malaysian J Pathol, 38, 257-66.

Adler D, de Bruyn F, Gray G, Allan BR, Williamson A-L (2008). Human Papillomavirus genotype distribution among Human Immunodeficiency Virus (HIV)-infected and Non- HIV-Infected women in Soweto, South Africa. J Clin Microbiol, 46, 4109-10.

Al-Awadhi R, Chehadeh W, Al-Jassar W, et al (2013). Viral load of human papillomavirus in women with normal and abnormal cervical cytology in Kuwait. J Infect Dev Ctries, 7, 130-6.

American Social Health Association (2012). Understanding Anogenital HPV: Infections, Diseases and Vaccines in Male and Female Patients.

Bosch FX, Burchell AN, Schiffman M, et al (2008). Epidemology and natural history of human papilloma infections and type specific implication in cervical neoplasia. Vaccine, 26, 1-16.

Clifford GM, Gallus S, Herrero R, et al (2005). Worldwide distribution of human papilloma virus types in cytologically normal women in the International Agency for Research on Cancer HPV prevalence surveys: a pooled analysis. Lancet, 366, 991-8.

Derese M (2010). Human Papillomavirus infection and genotype distribution in relation to cervical cytology abnormalities and HIV-1 infection in at Tikur Anbessa Teaching Hospital, AA, Ethiopia. Thesis, 90.

Donkoh ET (2016). Distribution of human papillomavirus genotypes and associated cervical disease in an unscreened population of women in Kunasai, Ghana. Thesis; 178.

Duvlis S, Plaseska-Karanfilska D, Bashevska N, et al (2001). A variant of human papillomavirus (HPV) type 66 is common among HPV-infected women from the Republic of Macedonia. Balkan J Med Genet, 4, 53- 7.

Asian Pacific Journal of Cancer Prevention, Vol 202063 
Faik A J, Saber MQ, Mohammed WJ, et al (2015). Genotyping of High-risk Human Papilloma virus (HPV) among Iraqi women in Baghdad by Multiplex PCR. J Biotechnol Res Center, 9, 38-45.

Hamlin-Douglas L (2008). Prevalence and determinants of human papillomavirus (HPV) infection in Inuit women of Nunavik, Quebec. Thesis Canada, 91.

Human Papillomavirus and Related disease reports, Fact Sheet. Iraq (2016). at www.hpvcentre.net.

Iraqi cancer registry (2012). Results of Iraqi cancer registry 1991-2010. World health organization, Ministry of Health (WHO), Baghdad, lraq.

Ismail AT, Ahmed NY, Hameed AA (2014). Prevalence of HPV immunostaining in benign, preneoplastic and neoplastic cervical lesions of kurdish women in Erbil City/Kurdistan of Iraq. Am J Res Commun, 2, 67-74.

Jeney C (2008). New molecular diagnostic approach of human papillomavirus detection: the role of experimental design in the development, analytical and clinical evaluation. Thesis, Budapest, 90 .

Levi JE, Fink MS, Canto CLM, et al (2002). Human papillomavirus prevalence, viral load and cervical intraepithelial neoplasia in HIV-infected women. Braz $J$ Infect Dis, 6, 129-35.

Megia L, Munoz D, Trueba G, Tinoco L, Zipata S (2016). Prevalence of human papilloma virus types in cervical cancerous and precancerous lesions in Ecuadorian women. J Med Virol, 88, 144-52.

Natphopsuk S, Ishida W, Sinawat S, Yuenyao P (2013). Human Papillomavirus genotypes and cervical cancer in Northeast Thailand. Asian Pac J Cancer Prev, 14, 6961-4.

Nayar R, Wilbur DC (2015). The Pap test and Bethesda 2014. Acta Cytologica, 59, 121-32.

Othman RT, Abdulljabar R, Saeed A, et al (2007). Cancer Incidence Rates in the Kurdistan Region/Iraq from 2007-2009.

Pity IS, Shamdeen MY, Wais SA (2012). Follow up of atypical squamous cell Pap Smears in Iraqi women. Asian Pac J Cancer Prev, 13, 3455-60.

Pity IS (2013). Pregnant and postpartum women with atypical glandular Cells, Follow-up and Evaluation for high-risk HPV. Cancer Res J, 1, 31-6.

Sauvageau C, Duval B, Gilca V, Lavoie F, Ouakki M (2007). Human papilloma virus vaccine and cervical cancer screening acceptability among adults in Quebec, Canada. BMC Public Health, 7, 304.

Schiffman M, Burk RD, Boyle S, et al (2015). A study of genotyping for management of Human Papillomaviruspositive, cytology-negative cervical screening results. J Clin Microbiol, 53, 52-9.

Schiffman M, Wentzensen N (2013). Human Papillomavirus infection and the multistage carcinogenesis of cervical cancer. Cancer Epidemiol Biomarkers Prev, 22, 553-60.

Verma I, Jain V, Kaur T (2014). Application of Bethesda system for cervical cytology in Unhealthy Cervi. J Clin Diagn Res, 8, 26-30.

Vink MA, Bogaards JA, van Kemenade FJ, et al (2013). Clinical progression of high-grade. Cervical Intraepithelial Neoplasia: Estimating the time to preclinical cervical cancer from Doubly Censored National Registry Data. Am J Epidemiol, 178, 1161-9.

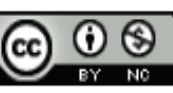

This work is licensed under a Creative Commons AttributionNon Commercial 4.0 International License. 